\begin{tabular}{|c|l|}
\hline Title & $\begin{array}{l}\text { Theoretical method for near-field Raman spectroscopy with multipolar Hamiltonian and real-time TDDFT : A pplication } \\
\text { to on- and off-resonance tip-enhanced Raman spectroscopy }\end{array}$ \\
\hline Author(s) & Takenaka, Masato; Taketsugu, Tetsuya; Iwasa, Takeshi \\
\hline Citation & $\begin{array}{l}\text { Journal of chemical physics, 154(2), 24104 } \\
\text { https://doi.org/10.1063/5.0034933 }\end{array}$ \\
\hline Issue Date & 2021-01-14 \\
\hline Doc URL & http://hdl.handle.net/2115/83818 \\
\hline Rights & $\begin{array}{l}\text { This article may be downloaded for personal use only. Any other use requires prior permission of the author and A IP } \\
\text { Publishing. This article appeared in J.Chem. Phys. 154, 024104 (2021) and may be found at } \\
\text { https://aip.scitation.org/doi full/10.1063/5.0034933. }\end{array}$ \\
\hline Type & article \\
\hline File Information & J. Chem. Phys. 1542_024104.pdf \\
\hline
\end{tabular}

Instructions for use 


\section{Theoretical method for near-field Raman spectroscopy with multipolar Hamiltonian and real-time-TDDFT: Application to on- and off-resonance tip-enhanced Raman spectroscopy}

Cite as: J. Chem. Phys. 154, 024104 (2021); https://doi.org/10.1063/5.0034933

Submitted: 26 October 2020 . Accepted: 20 December 2020 . Published Online: 08 January 2021

Masato Takenaka, (D) Tetsuya Taketsugu, and (D) Takeshi Iwasa

\section{ARTICLES YOU MAY BE INTERESTED IN}

Probing the deformation of [12]cycloparaphenylene molecular nanohoops adsorbed on metal surfaces by tip-enhanced Raman spectroscopy

The Journal of Chemical Physics 153, 244201 (2020); https://doi.org/10.1063/5.0033383

Tip-enhanced Raman spectroscopy for nanoscale probing of dynamic chemical systems The Journal of Chemical Physics 153, 170901 (2020); https://doi.org/10.1063/5.0027917

Model DFT exchange holes and the exact exchange hole: Similarities and differences The Journal of Chemical Physics 154, 024101 (2021); https://doi.org/10.1063/5.0031995

Challenge us. What are your needs for periodic signal detection?

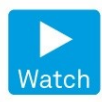

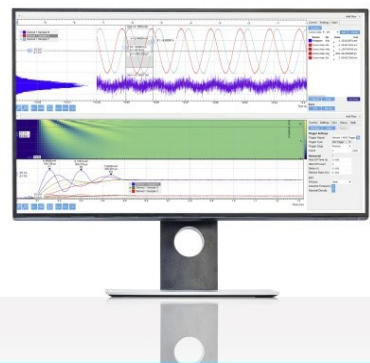

Zurich Instruments 


\title{
Theoretical method for near-field Raman spectroscopy with multipolar Hamiltonian and real-time-TDDFT: Application to on- and off-resonance tip-enhanced Raman spectroscopy
}

\author{
Cite as: J. Chem. Phys. 154, 024104 (2021); doi: 10.1063/5.0034933 \\ Submitted: 26 October 2020 - Accepted: 20 December 2020 - \\ Published Online: 8 January 2021 \\ Masato Takenaka, 'Tetsuya Taketsugu, ${ }^{2,3,4}$ (D) and Takeshi Iwasa ${ }^{2,3,4,5, a)}$ (iD)

\begin{abstract}
AFFILIATIONS
${ }^{1}$ Graduate School of Chemical Sciences and Engineering, Hokkaido University, Sapporo 060-0810, Japan

${ }^{2}$ Department of Chemistry, Faculty of Science, Hokkaido University, Sapporo 060-0810, Japan

${ }^{3}$ WPI-ICReDD, Hokkaido University, Sapporo 001-0021, Japan

${ }^{4}$ ESICB, Kyoto University, Kyoto 615-8245, Japan

${ }^{5}$ PRESTO, Japan Science and Technology Agency, Kawaguchi 332-0012, Japan
\end{abstract}

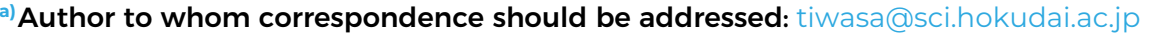

\begin{abstract}
Tip-enhanced Raman spectroscopy in combination with scanning tunneling microscopy could produce ultrahigh-resolution Raman spectra and images for single-molecule vibrations. Furthermore, a recent experimental study successfully decoupled the interaction between the molecule and the substrate/tip to investigate the intrinsic properties of molecules and their near-field interactions by Raman spectroscopy. In such a circumstance, more explicit treatments of the near field and molecular interactions beyond the dipole approximation would be desirable. Here, we propose a theoretical method based on the multipolar Hamiltonian that considers full spatial distribution of the electric field under the framework of real-time time-dependent density functional theory. This approach allows us to treat the on- and off-resonance Raman phenomena on the same footing. For demonstration, a model for the on- and off-resonance tip-enhanced Raman process in benzene was constructed. The obtained Raman spectra are well understood by considering both the spatial structure of the near field and the molecular vibration in the off-resonance condition. For the on-resonance condition, the Raman spectra are governed by the transition moment, in addition to the selection rule of off-resonance Raman. Interestingly, on-resonance Raman can be activated even when the near field forbids the $\pi-\pi^{*}$ transition at equilibrium geometry due to vibronic couplings originating from structural distortions.
\end{abstract}

Published under license by AIP Publishing. https://doi.org/10.1063/5.0034933

\section{INTRODUCTION}

The near field refers to the electromagnetic field near a surface. Localization of the near field has been utilized in molecular spectroscopy and microscopy to achieve a spatial resolution beyond the diffraction limit of the propagating light. Recently, chemical reactions induced by near field have been studied.
Near-field vibrational spectroscopy using a plasmon field has been widely adopted as a surface-enhanced vibrational spectroscopy method. IR spectroscopy using a plasmonic metal nanostructure is called surface-enhanced infrared spectroscopy (SEIRAS). In the case of Raman effects, it is called surface-enhanced Raman spectroscopy (SERS). ${ }^{6}$ Notably, because of the localized nature of the near field, the induced molecular excitation can be non-dipolar, 
which is different from the case with propagating light (plane wave).

Among near-field Raman spectroscopy techniques, we focus on tip-enhanced Raman spectroscopy (TERS), a type of surfaceenhanced vibrational spectroscopy using a metal tip. ${ }^{8-10}$ Localized surface plasmon resonance of a metal probe is accompanied by a strongly enhanced local electric field compared to the incident light. This local field induces dipole moment in a nearby molecule, and scattered light is detected as an enhanced Raman signal. There are two main experimental approaches for TERS. In atomic force microscope (AFM)-TERS, ${ }^{11}$ the AFM probe is coated with a thin film of plasmonic metal (e.g., $\mathrm{Au}$ and $\mathrm{Ag}$ ). ${ }^{12}$ The other is scanning tunneling microscope (STM)-TERS. In both techniques, an enhanced electric field is generated at the gap between the microprobe and the metal surface. ${ }^{13}$ The plasmonic field is not only strong but also spatially non-uniform at the molecular scale. Therefore, peaks of Raman-forbidden modes can be observed owing to the electric field gradient ${ }^{14}$ as is the case with SERS. ${ }^{15}$

One of the advantages of TERS is high spatial resolution in the Raman spectra and imaging. By scanning the tip over a molecule, the Raman spectrum can be obtained at each point, which allows one to map the Raman spectral intensities. A 15-nm resolution with ultrahigh vacuum (UHV)-TERS was reported in $2008 .{ }^{16}$ In a dry nitrogen environment at room temperature, a $1.7-\mathrm{nm}$ imaging resolution was reported for carbon nanotubes using STM-TERS. ${ }^{17}$ Recently, sub-molecular scale resolution ${ }^{18}$ and molecular vibrational mode dependency have been reported. ${ }^{19}$ In those experiments, either of the molecules is directly adsorbed onto the metal surface and/or the distance between the molecule and the metal tip is small. In such conditions, chemical interaction between the metal tip or substrate and the molecule may be important for the peak enhancement, as in the case of SERS.

Theoretical methods have been developed to understand the atomic resolution of TERS mapping. Jensen et al. developed a discrete interaction model/quantum mechanics (DIM/QM) method to calculate single-molecule TERS imaging maps. The effect of metal atoms in the tip is accounted for by classical atomic polarizability, while the molecule is modeled by quantum chemistry. ${ }^{20}$ Those authors also showed the importance of the electric field gradient in Raman spectra when the molecule was at the gap between the metal surface and the metal tip (modeled by a cluster). The dependence of the TERS signal of a single molecule on its vibrational modes was clarified. Recently, a locally integrated Raman polarizability density (LIRPD) method was proposed for understanding the mechanisms and selection rules of TERS. ${ }^{21}$ The method handles the Raman polarization density of the molecule and the nearfield distribution with a low computational cost, and it can qualitatively reproduce the computational results of the DIM/MM method. Luo et al. developed a computational method that considers the effect of spatially confined plasmons (SCPs) based on the gaugeinvariant Hamiltonian and reported that the SCP localized in a few angstroms can achieve atomic-scale resolution. ${ }^{22-25}$ Fiederling et al. investigated the quantum chemical effect for off- and onresonance TERS of a naphthalocyanine molecule, and the tip was modeled by the Ag atom/cluster. The excited states of the entire tip-molecule system were calculated by time-dependent density functional theory (TDDFT) ${ }^{26}$ The role of the charge-transfer state and intramolecular excitation state has been well studied for TERS imaging.

Conventional surface-enhanced spectroscopy is understood from both chemical and electromagnetic viewpoints. In recent experiments, the molecule can be decoupled from the surface and the tip by inserting an insulating $\mathrm{NaCl}$ thin film and positioning the STM tip away from the molecule, respectively. Such experiments have been performed to measure the molecule free from chemical effects. ${ }^{27}$ The insulator is inserted between the sample and substrate to decouple their electronic interaction. Recently, single-molecule STM-TERS using $\mathrm{NaCl}$ films was reported, and vibrational mode dependency was clearly observed. ${ }^{28}$ In that experiment, the energy of the incident light was tuned to the excited state, which has a transition dipole moment parallel to the substrate. Under this condition, signal enhancement by the electromagnetic field may become dominant. The electric field component parallel to the surface may play a role in the peak selectivity of the Raman spectrum, especially under the resonance condition.

Among these new experiments, we are interested in the intrinsic selection rule of a molecule excited by near field. We have previously studied the interaction between molecules and the near field using a multipolar Hamiltonian, which includes the infinite number of multipole interaction terms between the molecule and the electric field. For application to surface-enhanced vibrational spectroscopy, a computational method for near-field IR was developed. ${ }^{29,30}$ In the case of IR spectroscopy, one only needs to calculate the ground state of the molecule. However, Raman spectroscopy uses the scattering from molecules; therefore, the electronically excited states need to be calculated. Near-field electronic excitation was studied as a real-time electron dynamics of $\mathrm{NC}_{6} \mathrm{~N}$ with real-space real-time TDDFT. ${ }^{31}$ This method could be used to understand the new experiments because the spatial structure of the near field can be explicitly treated.

The current paper presents a computational method for Raman spectroscopy with non-uniform electric fields using real-time (RT)-TDDFT. The spatial distribution of the electric field is fully treated, and the peak intensity is calculated from the induced dipole moment. A method with RT-TDDFT has already been developed to compute Raman spectra under the dipole approximation. ${ }^{32}$ It was shown that the dipole polarizability under electron resonance conditions, calculated from the time propagation under linear perturbation conditions, is the same as that obtained using Jensen's polarizability method. ${ }^{33,34}$ The merit of RT-TDDFT is the computational efficiency ${ }^{33}$ and ability to achieve massive parallelization when in combination with the real space method. ${ }^{35}$ As an example, excited states of nanometer-scale gold clusters have been calculated with a massively parallel computation. ${ }^{36}$ In addition, the current approach can calculate both the resonance and non-resonance Raman spectra by only changing the energy of the incident field.

\section{THEORY}

The Raman signal is detected as scattered light generated from the induced dipole moment of a molecule. ${ }^{37}$ The intensity of the Raman spectra for the $k$ th normal mode $I_{k}$ is proportional to the square of the derivative of the induced dipole moment by normal coordinate, $Q_{k}$, 


$$
I_{k} \propto\left|\frac{\partial \boldsymbol{\mu}^{\text {ind }}(\omega)}{\partial \boldsymbol{Q}_{k}}\right|^{2},
$$

where $\omega$ is the frequency of the incident light. This formula assumes that the observer is located far from the molecule so that the higher multipole radiation can be neglected. Therefore, Eq. (1) may be used even when the molecule is irradiated by a complex electric field under typical experimental conditions. Meanwhile, the experimental conditions affect which component of the induced dipole moment produces the observed scattered light.

The frequency-dependent induced dipole moment $\mu^{\text {ind }}(\omega)$ is calculated by Fourier transform of the time-dependent induced dipole moment $\mu^{\text {ind }}(t)$,

$$
\boldsymbol{\mu}^{\text {ind }}(\omega)=\int \boldsymbol{\mu}^{\text {ind }}(t) e^{-i \omega t} d t
$$

$\boldsymbol{\mu}^{\text {ind }}(t)$ is derived from the time-dependent electron density,

$$
\begin{aligned}
& \boldsymbol{\mu}^{\text {ind }}(t)=\int \delta \rho(\boldsymbol{r}, t) \cdot \boldsymbol{r} d \boldsymbol{r}, \\
& \delta \rho(\boldsymbol{r}, t)=\rho(\boldsymbol{r}, t)-\rho(\boldsymbol{r}, 0),
\end{aligned}
$$

where $\rho$ is the electron density and $\delta \rho$ is its variation. The $\delta \rho$ induced by external fields can be obtained by solving the time-dependent Kohn-Sham equation as follows:

$$
\begin{gathered}
i \frac{\partial}{\partial t} \phi_{i}(\boldsymbol{r}, t)=\left(-\frac{\nabla^{2}}{2}+V_{\mathrm{H}}(\boldsymbol{r}, t)+V_{\mathrm{xc}}(\boldsymbol{r}, t)+V_{\mathrm{ext}}(\boldsymbol{r}, t)\right) \phi_{i}(\boldsymbol{r}, t), \\
\rho(\boldsymbol{r}, t)=\sum_{i=1}^{n}\left|\phi_{i}(\boldsymbol{r}, t)\right|^{2},
\end{gathered}
$$

where $\phi_{i}$ is the Kohn-Sham orbital, $V_{\mathrm{H}}$ is the Hartree potential, $V_{\mathrm{xc}}$ is the exchange-correlation potential, and $V_{\text {ext }}$ is the external potential. By using the external potential, which includes the interaction between the molecule and the non-uniform electric field, the induced dipole moment can be obtained. In this study, we used a multipolar Hamiltonian that considers all the multipolar interactions between the molecule and the external electric field. This Hamiltonian is a canonical transformation of the minimal coupling Hamiltonian. ${ }^{38}$ Briefly, the transformation is done as $H_{\text {mult }}=\exp (-i S) H_{\min } \exp (i S)$, where $S=(1 / \hbar) \int \boldsymbol{P}^{\perp}(\boldsymbol{r}) \cdot \boldsymbol{A}(\boldsymbol{r}) d \boldsymbol{r}, \boldsymbol{P}^{\perp}$ is the transverse component of the electric polarization field operator of a molecule, and $\boldsymbol{A}$ is the vector potential. ${ }^{38}$ It should be noted that in our study, all the magnetic interactions are omitted, and only the electric interactions are considered. Then, we will have the following interaction Hamiltonian:

$$
V_{\mathrm{ext}}(\boldsymbol{r}, t)=-\int \hat{\boldsymbol{P}}\left(\boldsymbol{r}^{\prime}, t\right) \cdot \boldsymbol{E}\left(\boldsymbol{r}^{\prime}, t\right) d \boldsymbol{r}^{\prime},
$$

where $\hat{\boldsymbol{P}}$ is the electric polarization field, and its explicit form is

$$
\boldsymbol{P}(\boldsymbol{r})=\sum_{\alpha} e_{\alpha}\left(\hat{\boldsymbol{q}}_{\alpha}-\boldsymbol{R}\right) \int_{0}^{1} d \lambda \delta\left[\boldsymbol{r}-\boldsymbol{R}-\lambda\left(\hat{\boldsymbol{q}}_{\alpha}-\boldsymbol{R}\right)\right],
$$

where $\mathrm{e}_{\alpha}$ and $\hat{\boldsymbol{q}}_{\alpha}$ are the charge and position operator of the $\alpha$ th electron in the molecule, respectively. The Taylor expansion of this electric polarization field gives multipoles such as dipoles, quadrupoles, and octapoles.

In sharp contrast to the dipole approximation aided by higher multipoles, here, we use the above original form of the electric polarization field without any truncation. This means that all orders of the multipole are considered. For the DFT calculation, the multipolar potential is defined by the following formula: ${ }^{31}$

$$
\begin{gathered}
V_{\mathrm{ext}}(\boldsymbol{r}, t)=(\boldsymbol{r}-\boldsymbol{R}) \cdot \boldsymbol{E}_{\mathrm{eff}}(\boldsymbol{r}, t), \\
E_{\mathrm{eff}}(\boldsymbol{r}, t)=\int_{0}^{1} d \lambda \boldsymbol{E}[\boldsymbol{R}+\lambda(\boldsymbol{r}-\boldsymbol{R}), t],
\end{gathered}
$$

where $\boldsymbol{R}$ is the center of mass of the molecule. The integration of $\lambda$ comes from the definition of the electric polarization field. In Eq. (9), a spatially non-uniform electric field obtained numerically by solving Maxwell's equations can be used as the external field. For example, an electric near-field around a silver nanoplate was used in our previous study to obtain near-field IR spectra. ${ }^{30}$

Before going to the next chapter, we would like to mention on the multipolar expansion. We rely on the above-mentioned framework, which begin by the Taylor expansion of charge density, while the other way can be the expansion of the non-uniform field itself. ${ }^{39}$ They give the same result. The expansion of the charge distribution includes the derivative on Dirac's delta function. By integrating Eq. (7) by parts, the derivative on the delta function moves to the electric field, and then, the form will look the same. The use of the Taylor series of charge distribution for the interaction Hamiltonian can then lead to multipolar magnetic interactions in the full multipolar Hamiltonian (this is not included in our paper). ${ }^{38}$ For both frameworks, the first interaction is a point charge and scalar potential, the next term is a dipole and electric field, and then quadrupole moment and field gradient. The use of lower orders of multipole interactions works for the slowly varying field. In the near-field case, however, the field has a very sharp intensity decrease, and the distribution of the electric field vectors is not uniform or even radially distributed, as shown in Fig. 2. For such cases, using all the multipolar interactions up to infinite orders [i.e., using Eq. (8)] would be convenient compared to the adding higher-order multipoles one by one to the dipole interaction because the convergence with finite orders of multipole interactions is not guaranteed, and then, an multipole of infinite order may be necessary.

\section{COMPUTATIONAL DETAILS}

\section{A. Computational scheme}

First, geometry optimization and normal mode analysis are performed for the molecule in the electronic ground state. Next, excited-state calculations are performed with the real-time propagation method. The time variation of the induced dipole moments is calculated for distorted geometries, where the coordinate of one atom is slightly displaced in each of the $x, y$, and $z$ directions. Third, Fourier transformation is applied to the induced dipole moment to obtain its counterpart in the frequency domain. Fourth, the derivatives of $\boldsymbol{\mu}^{\text {ind }}$ with respect to the atomic Cartesian coordinates are 
calculated and then transformed from Cartesian coordinates to normal coordinates using the transformation matrix $L$ obtained by normal mode analysis. Finally, the Raman spectrum is obtained from the square of the absolute value of the normal coordinate derivatives of $\mu^{\text {ind }}$

\section{B. DFT and RT-TDDFT}

All the main electronic structure calculations were performed with the real-time and real-space TDDFT program Octopus 9.2. ${ }^{40}$ The Perdew-Burke-Ernzerhof (PBE) density functional ${ }^{41,42}$ and the SG15 Optimized Norm-Conserving Vanderbilt (ONCV) pseudopotential $^{43}$ were used. Instead of the more widely used basis sets, Octopus adopts a real-space grid technique. The computational space is made up by the superposition of spheres (radius $7.0 \AA$ ) around the atoms. The mesh size of the computational space was $0.15 \AA$.

Normal mode analysis was performed using the finite difference method as implemented in Octopus. The default parameterization was used in which the gradients are obtained when the atomic coordinates are shifted by 0.01 Bohr $(\sim 0.00529 \AA)$.

For excited states, real-time electron dynamics calculations were performed. The time step of the real-time calculation was $0.0015 / \mathrm{eV}(\sim 0.99 \mathrm{as})$, and the total number of computational steps was 30000 .

The absorption spectra were calculated using a $\delta$ pulse method. ${ }^{44}$ In this method, the ground state wavefunction perturbed by $e^{(i \delta \boldsymbol{k} \cdot \boldsymbol{r})}(|\delta \boldsymbol{k}|=0.01 \AA$ ) was used as an initial wavefunction for realtime propagation with the molecular Hamiltonian (i.e., without any external fields).

For Raman spectra, the real-time calculation under the dipole approximation was performed by applying the uniform electric field $\boldsymbol{E}$. The electric-field amplitude of uniform electric fields is set to 3.8 $\times 10^{-6}$ a.u. $\left(\sim 2.4 \times 10^{10} \mathrm{~W} / \mathrm{cm}^{2}\right)$. For near-field excitation, the realtime propagation was performed under the external potential given by Eq. (9) generated by a point dipole, as described in Sec. III C. We assume that this electric field oscillates at a frequency of $\omega$. The envelope function $\phi$ is multiplied by the potential for numerical stabilization,

$$
\phi(t)=\cos \left(\frac{\pi}{2} \cdot \frac{t-3 t_{0}}{t_{0}}\right)
$$

A pulse duration of $t_{0}=620$ a.u. ( $\left.~ 30 \mathrm{fs}\right)$ is used in our calculation.

To analyze the electronic excitations, we calculated the Fourier transform of the electronic density as follows:

$$
\delta \rho(\boldsymbol{r}, \omega)=\int \delta \rho(\boldsymbol{r}, t) e^{-i \omega t} d t
$$

In the weak perturbation regime, the induced electron density is low (i.e., the mixing of excited states to the ground state wavefunction is very small). For such cases, the electron density difference can be approximated by the transition density. ${ }^{45,46}$ The transition densities obtained in our method were compared with the TURBOMOLE results to assign the induced excited states.

\section{Near field model}

In this study, we calculated the TERS spectrum of benzene as an example. The near field around the metal tip is modeled by the near field of a point dipole expressed by the following equation:

$$
\boldsymbol{E}^{\mathrm{nf}}(\boldsymbol{r})=\frac{[3 \boldsymbol{n}(\boldsymbol{n} \cdot \boldsymbol{\mu})-\boldsymbol{\mu}]}{4 \pi \varepsilon_{0} \boldsymbol{r}^{3}},
$$

where $\boldsymbol{n}$ is the normalized coordinate vector and $\boldsymbol{\mu}$ is the dipole moment. The effect of the metal surface can be neglected as we focus on the experimental setup used in our previous paper ${ }^{28}$ in which the molecule is decoupled from the metal substrate by a $\mathrm{NaCl}$ thin film, as shown in Fig. 1(a).

From Fig. 1(b), the point dipole is located $5 \AA$ above the benzene, and its strength is $1 \mathrm{D}$. The electric field distribution around the benzene is also shown in Figs. 2(a)-2(d). The $x$ and $y$ components of the electric field have a ring-like intensity distribution, and the electric field vectors spread radially. The $z$ component of the electric field radiates from the point dipole, and its intensity decreases with increasing distance from the dipole. This electric field distribution is qualitatively consistent with that calculated using the finite element method for the simulation of the TERS experiment. ${ }^{47}$ The maximum intensity ratio of the $x, y$, and $z$ components on the $x y$-plane is $\sim 1: 1: 2$.

In the previous experimental STM-TERS study, ${ }^{28}$ the tipmolecule distance was set to $5.8 \AA$, in contrast to Apkarian's STM-TERS, where the distance is smaller than $2 \AA .{ }^{19}$ It was shown that with this larger tip-molecule distance, the intrinsic vibrational modes of the target molecule are rather unaffected, suggesting that chemical interactions (charge transfer and other perturbations) can be very weak or even ruled out. In the present model, the distance is not exactly the same as that experiment, but we believe that in the present distance, the chemical effect may not be dominant.

Numerical integration of Eq. (10) is done by

$$
E_{\mathrm{eff}}(\boldsymbol{r}, \boldsymbol{R})=\sum_{i=0}^{M} \frac{1}{M} \boldsymbol{E}\left(\boldsymbol{R}+\frac{i}{M} \boldsymbol{r}\right),
$$

where $\boldsymbol{R}$ is the center of gravity of the molecule. The points for calculating the electric field are independent of the grid for DFT calculations. In this study, we used $M=100$. When we computed the Raman spectra for $M=30,60,100,150$, the peak distribution (a)

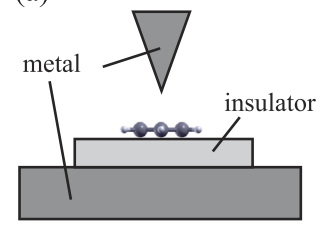

(b)

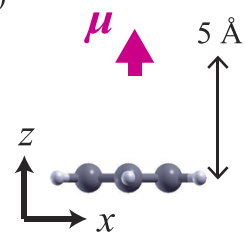

FIG. 1. (a) Scheme of the experimental setup in which the insulator decouples the metal and benzene molecule. (b) The computational model in which the point dipole is placed above the benzene and the origin is set at the center of benzene. 
(a)
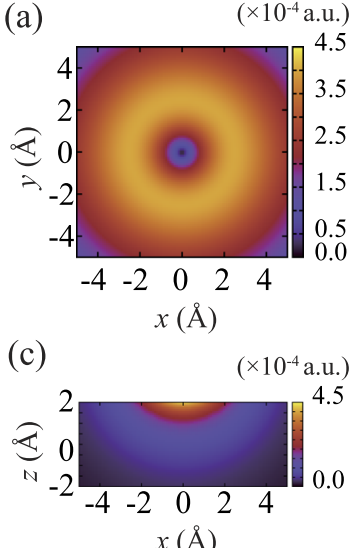

(b)

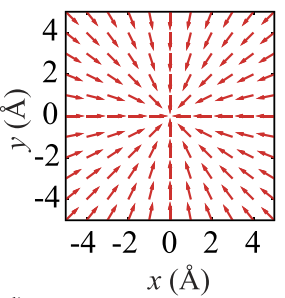

(d)

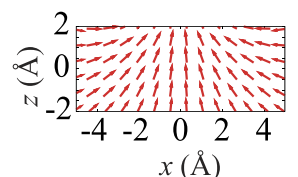

$x(\AA)$

FIG. 2. (a) Intensity map of $\left(E_{x}^{2}+E_{y}^{2}\right)^{1 / 2}$ on the $x y$-plane. (b) Normalized electric field vectors on the $x y$-plane. (c) Intensity map of $\left(E_{x}^{2}+E_{z}^{2}\right)^{1 / 2}$ on the xz-plane. (d) Normalized electric field vectors on the $x z$-plane.

was almost unchanged and converged when the value of $M$ became large. In this computational setup for benzene, the computational grids exist within $10 \AA$ from the origin $\boldsymbol{R}$. Then, the largest step size is $0.33 \AA$ when $M=30$ and $0.1 \AA$ when $M=100$.

\section{Raman spectra}

To obtain the Raman spectrum, we need the normal coordinate derivatives of the induced dipole moment $\boldsymbol{\mu}^{\text {ind }}$, which is obtained from the Cartesian coordinate derivative of $\boldsymbol{\mu}^{\text {ind }}$.

The normal coordinate derivative of the induced dipole moment in Eq. (1) is transformed from the Cartesian coordinate derivative by the transform matrix $L$, which is obtained by the normal mode analysis described above,

$$
\frac{\partial \boldsymbol{\mu}^{\text {ind }}(\omega)}{\partial Q_{i}}=\sum_{j=1}^{3 N} L_{j i} \frac{\partial \boldsymbol{\mu}^{\text {ind }}(\omega)}{\partial x_{j}},
$$

where $N$ is the number of atoms in the molecule and $x_{j}$ is the Cartesian displacement of atoms in the $x, y$, and $z$ directions.

The Cartesian coordinate derivative in Eq. (15) is evaluated by

$$
\frac{\partial \boldsymbol{\mu}^{\text {ind }}(\omega)}{\partial x_{j}}=\frac{\boldsymbol{\mu}^{\text {ind }}(\omega)_{x_{j}+\Delta x / 2}-\boldsymbol{\mu}^{\text {ind }}(\omega)_{x_{j}-\Delta x / 2}}{\Delta x} .
$$

\section{BENCHMARK TEST}

In order to check the accuracy of the present computational scheme and parameterization, we compared our calculated absorption spectra and off-resonance Raman spectra under the dipole approximation with those calculated by TURBOMOLE ${ }^{48}$ at the level of PBE/aug-cc-pVDZ ${ }^{49}$ under the resolution of the identity approximation. The use of large basis sets in TURBOMOLE is meant to account for the Rydberg character of the excited states, while the

real-space grid techniques naturally describe the Rydberg character in Octopus with the present parameterizations.

Figure 3 shows the absorption spectrum of benzene. The excited states allowed for $x$ and $y$ are labeled $\mathrm{e}_{1 \mathrm{u}}$ and for $z$ are labeled $\mathrm{a}_{2 \mathrm{u}}$. The absorption spectra are almost quantitatively the same between the Octopus and TURBOMOLE calculations, while the absorption peak of $e_{1 u}$ is split for Octopus results. The calculations in Octopus do not use symmetry. Thus, $x$ and $y$ are not exactly the same in the real-space technique.

To assign the lowest-energy excited states, we compare the electron density difference obtained by Octopus with the transition density obtained by TURBOMOLE. The transition density is defined as the weighted sum of the product of occupied and virtual orbitals that form the excited state. The transition density is reported to correspond to the imaginary part of the Fourier-transformed electron density difference. ${ }^{4}$

The Kohn-Sham orbitals related to the lowest energy transitions for $\mathrm{a}_{2 \mathrm{u}}$ and $\mathrm{e}_{1 \mathrm{u}}$ are shown in Fig. 4(a), and the corresponding transition densities are shown in Fig. 4(b). These data are compared with the Fourier transform of the electron density difference obtained by Octopus in the real-time propagations with the $\delta$ pulse and uniform electric fields, as shown in Figs. 4(c) and 4(d). Apparently, the shapes of transition densities in Figs. 4(b)-4(d) are almost the same as the Fourier transformed densities. By comparison, the lowest energy excited state for $x / y$ is the $\pi-\pi^{*}$ transition, and that for $z$ is termed $\pi$ to Rydberg $\sigma^{*}$ transitions.
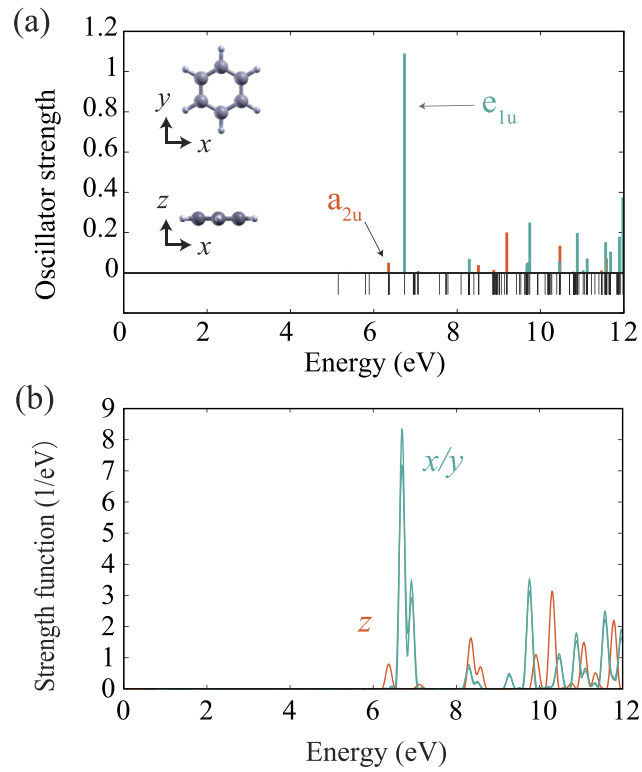

FIG. 3. (a) Oscillator strength of benzene calculated by TURBOMOLE. The orange and green lines show peaks of the $a_{2 u}$ and $e_{1 u}$ excitations, respectively. The black lines under the spectrum indicate the positions of all excited states. (b) Strength function of Octopus from applying an impulsive force in either the $x, y$, or $z$ directions. The peak area of the strength function corresponds to the oscillator strength. 
(a)

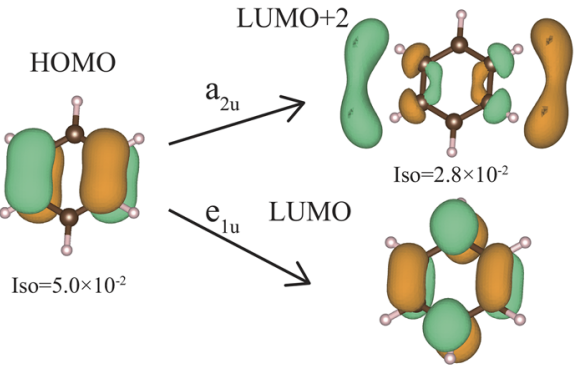

Iso $=5.0 \times 10^{-2}$

(b) $\mathrm{a}_{2}$

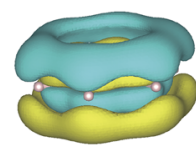

Iso $=2.0 \times 10^{-4}$

(c) $\mathrm{z}$

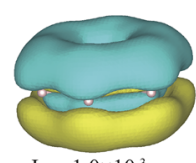

Iso $=1.0 \times 10^{-3}$

(d) $\mathrm{z}$

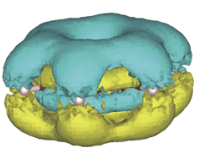

Iso $=1.0 \times 10^{-5}$ $e_{1 u}$

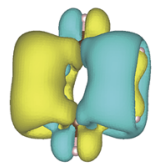

Iso $=1.0 \times 10^{-3}$

X

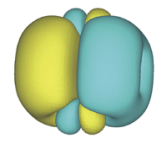

Iso $=1.0 \times 10^{-3}$

$\mathrm{X}$

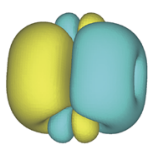

Iso $=1.0 \times 10^{-4}$

FIG. 4. (a) Kohn-Sham orbitals obtained with TURBOMOLE concerning the lowest energy $a_{2 u}$ and $e_{1 u}$ excitations. These orbitals are doubly degenerate, and only one of them is shown for simplicity. (b) Transition densities of the lowest excited states of $\mathrm{a}_{2 u}$ and $\mathrm{e}_{1 \mathrm{u}}$ symmetries obtained with TURBOMOLE. The yellow and blue surfaces represent positive and negative components, respectively. (c) Fourier-transformed electron density differences triggered by an impulsive force, obtained from RT-TDDFT calculations using Octopus. Left: $\omega=6.36 \mathrm{eV}$, force in the $z$ direction. Right: $\omega=6.70 \mathrm{eV}$, force in the $x$ direction. (d) Fourier-transformed electron density differences induced by a uniform electric field at the electronic excitation energies, obtained by RT-TDDFT calculations using Octopus. Left: $\omega=6.36 \mathrm{eV}$, electric field in the $z$ direction. Right: $\omega=6.70 \mathrm{eV}$, electric field in the $x$ direction.

In the present calculation, we use generalized gradient approximation functional, which is known to underestimate the excitation energy, especially for extended states such as Rydberg states. To recover some of the deficiencies, a range separate or long-range corrected (LC) DFT would be required. As for the size of the basis sets, we used a real-space grid that can be considered to use very large basis sets and we consider that the spatial distribution of Rydberg states can be described fairly well. In addition, Lorentzon et al. reported that the oscillator strength obtained with CASSCF for this state is 0.052 , which is very close to our TDDFT (TURBOMOLE) result (0.050). ${ }^{51}$ Based on these considerations, the present TDDFT calculations give qualitatively acceptable transition dipole moments and thus the Raman spectra. It is still very important, however, to clarify the use of TDDFT compared with a highly accurate post-Hartree-Fock method, but this would require very

efficient computational algorithm, and this would be the future challenge.

Figure 5 compares the off-resonance Raman spectra obtained with TURBOMOLE and our method. For comparison, the rotational average was taken for the off-resonance Raman spectra calculated with Octopus. Under this condition, the Raman signal of the $i$ th mode is written as follows: ${ }^{52}$

$$
\begin{gathered}
S_{i}=45\left(\frac{\partial a}{\partial Q_{i}}\right)^{2}+7\left(\frac{\partial \gamma}{\partial Q_{i}}\right)^{2}, \\
a=\frac{1}{3}\left(\alpha_{x x}+\alpha_{y y}+\alpha_{z z}\right), \\
\gamma^{2}=\frac{1}{2}\left[\left(\alpha_{x x}-\alpha_{y y}\right)^{2}+\left(\alpha_{y y}-\alpha_{z z}\right)^{2}+\left(\alpha_{z z}-\alpha_{x x}\right)^{2}\right. \\
\left.+6\left(\alpha_{x y}^{2}+\alpha_{y z}^{2}+\alpha_{z x}^{2}\right)\right],
\end{gathered}
$$

where $\alpha$ is the polarizability tensor, $a$ is the isotropic polarizability, and $\gamma$ is the anisotropic polarizability. The off-resonance Raman spectra were obtained by using the dynamic polarizability at $3 \mathrm{eV}$ by TURBOMOLE. ${ }^{53}$ In our approach, the $i j$ component of the polarizability tensor $\alpha_{i j}$ can be calculated by

$$
\alpha_{i j}(\omega)=\frac{\mu_{i}^{\text {ind }}(\omega)}{E_{j}},
$$

where $E_{j}$ is the electric-field strength. For comparison, the normal Raman spectra under the dipole approximation are shown at the top of Fig. 5. The vertical axis represents the normalized Raman activity. As these off-resonance Raman spectra match very well, we consider the results of our calculations to be reliable.
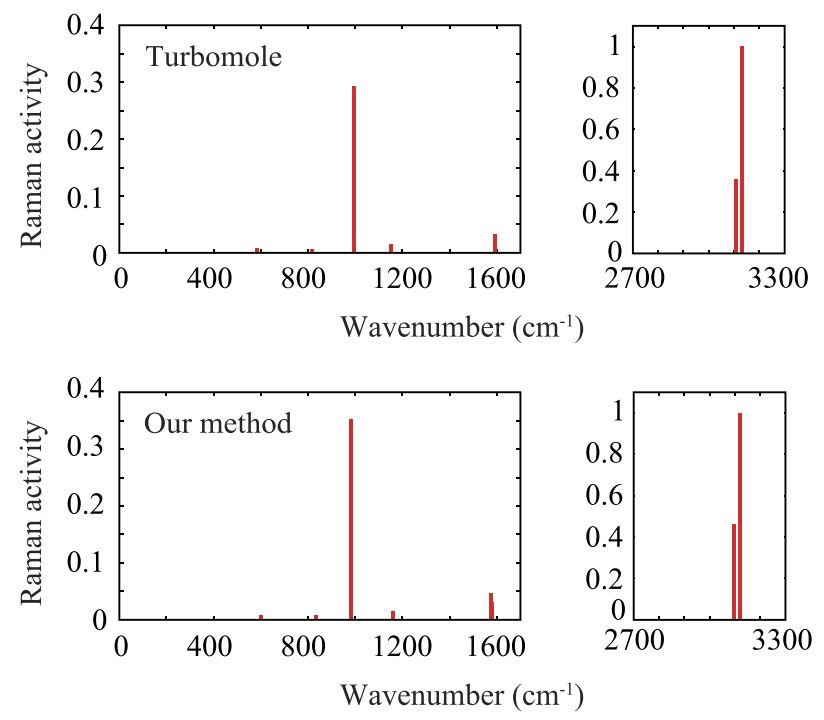

FIG. 5. Normalized Raman activity of benzene calculated by TURBOMOLE and our method. 


\section{RESULTS AND DISCUSSION}

Here, we discuss the Raman spectra obtained using our approach, with a particular focus on the near-field excitations. The Raman spectra in the range of $0 \mathrm{~cm}^{-1}-1700 \mathrm{~cm}^{-1}$ and $2700 \mathrm{~cm}^{-1}$ $3300 \mathrm{~cm}^{-1}$ are shown in Fig. 6, along with the corresponding vibrational modes. The normal Raman spectra are the same as those shown in Fig. 5(b), but convoluted with the Lorentz function. For the near-field Raman spectra, the off-resonance and on-resonance conditions were considered. The near-field frequency of $3.0 \mathrm{eV}$ was used for the off-resonance condition and $6.36 \mathrm{eV}$ and $6.70 \mathrm{eV}$ for the on-resonance conditions. Compared with the peak intensities of off-resonance Raman, those of on-resonance Raman at $6.36 \mathrm{eV}$ and $6.70 \mathrm{eV}$ are enhanced by about 100 times. In addition, the two onresonance Raman spectra show different peak distributions. The $x$, $y$, and $z$ contributions to $\left|\frac{\partial \mu^{\text {ind }}(\omega)}{\partial Q_{k}}\right|^{2}$ are separately shown in Fig. 7 for analysis.

\section{A. Off-resonance Raman}

The peak selectivity under the off-resonance condition in Figs. 6(c) and 6(d) is explained by molecular vibrational symmetry and spatial structure of the near field.
Let us first focus on the Raman-forbidden modes in far-field Raman, which become active in near-field Raman. The vibrational mode b shown in Fig. 6 is Raman-forbidden because the normal coordinate derivative of the dipole polarizability is zero at the equilibrium structure. However, this mode is active and shows a peak in the near-field Raman spectra [Figs. 6(c) and 6(g)]. From the decomposition in Fig. 7(a), this peak originates from the $z$ component since the near field is not uniform, as shown in Fig. 8(a). In this condition, the normal coordinate derivative of the electric field and thus the induced dipole moment become nonzero at the equilibrium structure, $Q=0$. The in-plane modes $\mathbf{f}$ and $\mathbf{i}$ are forbidden for the same reason as for mode $\mathbf{b}$ in the far-field Raman spectra. These modes also become active in near-field Raman because $E_{x}^{\text {nf }}$ and $E_{y}^{\text {nf }}$ are non-uniform, which causes the normal coordinate derivative of the induced dipole moment to be nonzero at the equilibrium structure. In the near-field excitation, the induced dipole moment depends on the coupling between the higher-order polarizability and higher-order derivative of the electric field, as shown in the following equation:

$$
\mu_{i}^{\text {ind }}=\sum_{j} \alpha_{i j} E_{j}+\sum_{j, k} \frac{1}{3} A_{i j k} \frac{\partial E_{j}}{\partial k}+\cdots .
$$

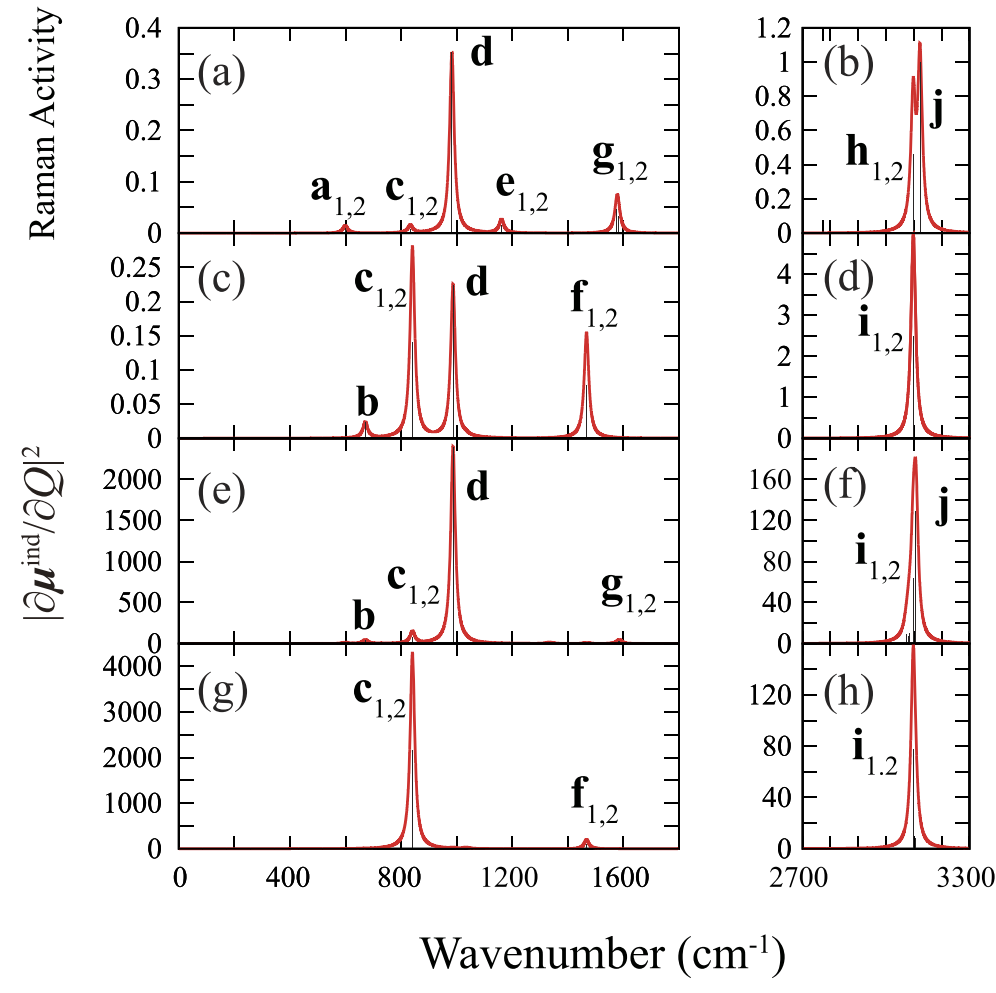

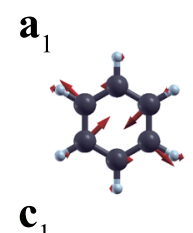

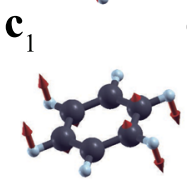

$\mathbf{a}_{2}$
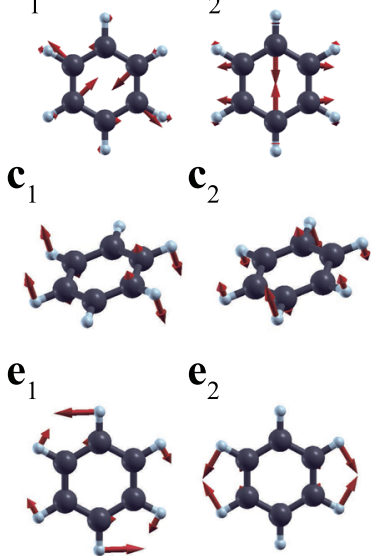

$\mathbf{e}_{2}$
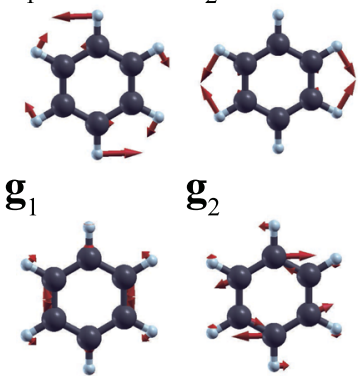

$\mathbf{g}_{2}$
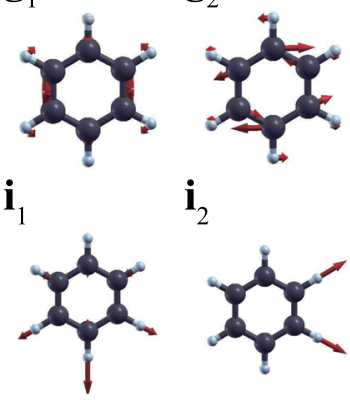

$\mathbf{i}_{2}$

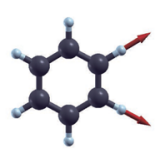

b

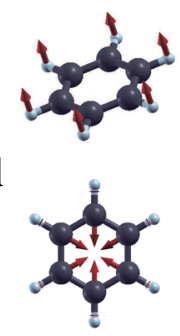

$\mathbf{f}_{1} \quad \mathbf{f}_{2}$

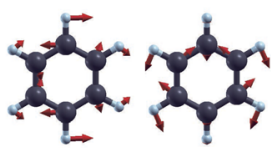

$\mathbf{h}_{1} \mathbf{h}_{2}$
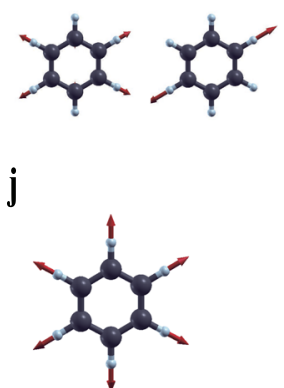

FIG. 6. (a) and (b) Normalized far-field Raman activity of benzene. Near-field Raman spectra of benzene under the (c) and (d) off-resonance frequency condition (3.00 eV) and on-resonance conditions of $(\mathrm{e})$ and $(\mathrm{f}) 6.36 \mathrm{eV}$ and $(\mathrm{g})$ and $(\mathrm{h}) 6.70 \mathrm{eV}$. The vibration modes labeled in the spectra are shown in the right. 

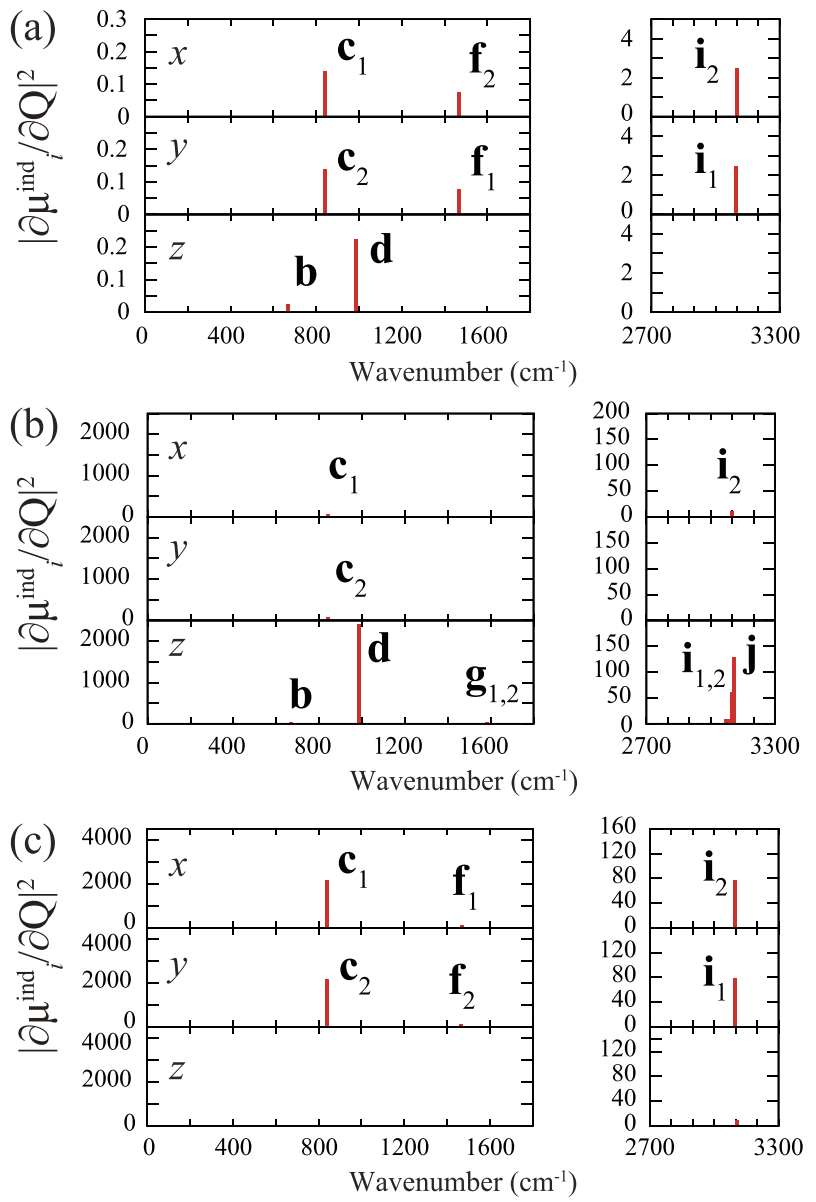

FIG. 7. Decomposition of the near-field Raman activities shown in Figs. $6(\mathrm{c})-6(\mathrm{~h})$. The $x, y$, and $z$ components of $\left|\frac{\partial \mu^{\text {ind }}(\omega)}{\partial Q}\right|^{2}$ with near-field frequencies at (a) $3.00 \mathrm{eV}$ (off-resonance), (b) $6.36 \mathrm{eV}$ ( $\mathrm{a}_{2 u}$ resonance), and (c) $6.70 \mathrm{eV}$ ( $\mathrm{e}_{1 \mathrm{u}}$ resonance). The peak labels are the same as in Fig. 6.

In the SERS study on the gradient-induced breaking of the selection rule, ${ }^{15,54,55}$ quadrupole interaction was considered to explain the missing Raman peaks under the dipole approximation. Again, all these multipole interactions are included in our current calculation.

Next, we discuss the vibrational modes that are active in both far- and near-field Raman but with different origins. In far-field Raman, the peak $\mathbf{c}_{1}$ consists of $x z$ and $y z$ components in the polarizability derivative tensor. Therefore, all the directions of $\boldsymbol{\mu}^{\text {ind }}$ are concerned. On the other hand, in near-field Raman, only $\mu_{x}^{\text {ind }}$ is concerned, as shown in Fig. 7(a). The motion of $\mathrm{H}$ atoms in the $\mathbf{c}_{1}$ mode is anti-symmetric in the $z$ direction, as shown in Fig. 8(b). Together with the near-field distribution in Fig. 8(b), the direction of induced dipole moment is inverted by the $\mathbf{c}_{1}$ vibration, and thus, $\partial \mu_{x}^{\text {ind }} / \partial Q$ is nonzero. The same explanation holds for $\mathbf{c}_{2}$ mode and $E_{y}^{\mathrm{nf}}$. The difference in the origin is prominent for
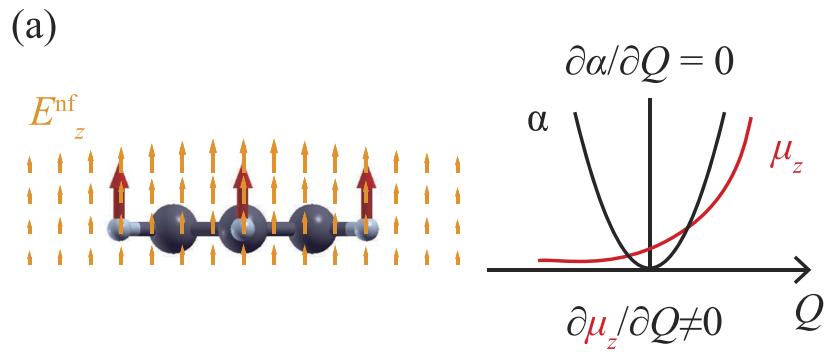

(b)

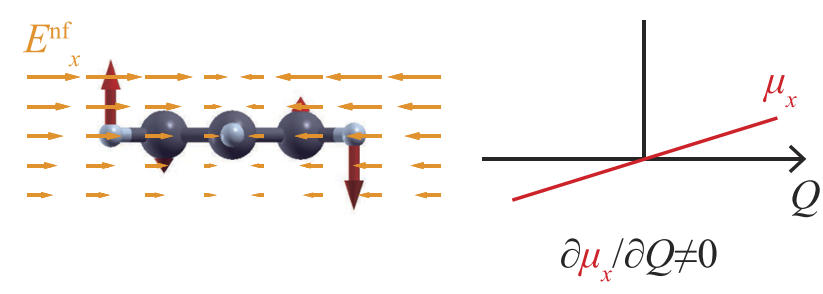

FIG. 8. (a) The scheme of vibrational mode $\mathbf{b}$ and the $E_{z}^{\mathrm{nf}}$, as well as the change of polarizability (black curve) and induced dipole moment (red curve) as a function of the normal coordinate. (b) The same for mode $c_{1}$ and $E_{x}^{\mathrm{nf}}$.

peak $\mathbf{d}$. This is the breathing mode of the carbon ring. In farfield Raman, the peak is derived from the $x x$ and $y y$ components of the polarizability. In near-field Raman, the $E_{x}^{\mathrm{nf}}$ and $E_{y}^{\mathrm{nf}}$ are distributed radially and cannot induce dipole moment within the $x y$-plane, as shown in Fig. 9. Thus, $\mu_{x / y}^{\text {ind }}$ does not contribute to peak $\mathbf{d}$. In near-field Raman, peak $\mathbf{d}$ comes from $\mu_{z}^{\text {ind }}$ since $E_{z}^{\mathrm{nf}}$ is non-uniform, as shown in Fig. 9. These things are clearly shown in Fig. 7(a).

Finally, we discuss the peaks that are present in far-field Raman spectra but absent in the near-field Raman spectra, namely, modes $\mathbf{a}_{1,2}, \mathbf{e}_{1,2}, \mathbf{g}_{1,2}, \mathbf{h}_{1,2}$, and $\mathbf{j}$. Except for $\mathbf{j}$, all these vibrational modes belong to the $E_{2 g}$ symmetry. In the far-field spectra, contributions to these Raman signals come from the $x x-y y$ and $x y$ components of the polarizability derivative tensors, whereas the contributions from
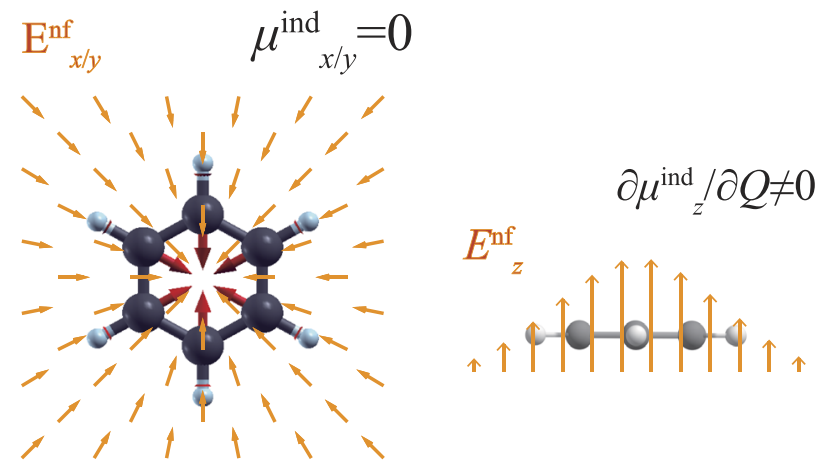

FIG. 9. Schematic top and side views of the vibrational mode $\mathbf{d}$ and the near field. 
$z z, x z$, and $y z$ are zero. According to our method, the signal consists of $\mu_{x}^{\text {ind }}$ and $\mu_{y}^{\text {ind }}$. The present near field is radially distributed in the $x y$-plane, and therefore, these contributions are zero. Although the near field is non-uniform in the $z$ direction, the contribution from $\mu_{z}^{\text {ind }}$ still seems to be very weak. As for mode $\mathbf{j}$, its far-field Raman signal consists of $\mu_{x}^{\text {ind }}$ and $\mu_{y}^{\text {ind }}$, while its near-field Raman signal consists of $\mu_{z}^{\text {ind }}$ as in mode d. However, compared to mode d, it seems that $\mathrm{C}-\mathrm{H}$ stretching has little effect on the induced dipole, and the peak intensity is very weak. In short, the "missing" peaks in the nearfield Raman are not forbidden but merely very weak because the contributing components change between far-field and near-field illuminations.

\section{B. On-resonance Raman}

Compared to off-resonance near-field Raman, one more thing should be considered in resonance near-field Raman to understand the selection rule, namely, the nature of the excited electronic state involved. Here, we applied the near field at the frequencies of $6.36 \mathrm{eV}$ and $6.70 \mathrm{eV}$, which are the excitation energies of the $\mathrm{a}_{2 \mathrm{u}}$ and $e_{1 u}$ excited states, respectively. The Raman spectra shown in Figs. 6(e)-6(h) are clearly different from the off-resonance ones. Furthermore, these spectra clearly depend on the frequency of the near field, suggesting that the involved excited electronic states are different. Figures 6(e) and 6(f) are spectra obtained with the near field at $6.36 \mathrm{eV}$, which can excite the lowest $\mathrm{a}_{2 \mathrm{u}}$ excited state. On the other hand, the spectra in Figs. 6(g) and 6(h) are obtained with the near field at $6.70 \mathrm{eV}$, and they are resonance Raman due to a vibronic coupling. At the equilibrium geometry, the molecule has $\mathrm{D}_{6 h}$ symmetry, and the near field in the $x y$-plane is radially distributed. Therefore, the $\mathrm{e}_{1 \mathrm{u}}\left(\pi-\pi^{*}\right)$ state cannot be excited by the near field. When the molecule vibrates, the symmetry decreases, and the $\pi-\pi^{*}$ transition can be occurred.

Let us first discuss the near-field Raman at the frequency of $6.36 \mathrm{eV}$. The corresponding electron density difference and Fourier transform of the induced dipole moment are shown in Figs. 10(a) and $10(\mathrm{c})$. Comparing them to Figs. 3 and 4 , the lowest energy $\mathrm{a}_{2 \mathrm{u}}$ excited state seems to be excited. The asymmetry above and below the benzene ring plane originates from the inhomogeneous near field intensity, as the field distribution becomes wider away from the tip, as shown in Fig. 2(d). In this resonant condition, peak d is the strongest. This peak arises from $\mu_{z}^{\text {ind }}$ instead of $\mu_{x / y}^{\text {ind }}$ for the same reason as in the off-resonance condition (discussed above). In resonance Raman, molecular orbitals concerning the chosen excited state affect the peak selectivity. When the spatial overlap between the molecular orbitals and vibrational mode is large, the change in the induced dipole may also be large, giving rise to a large Raman signal. Under the near field at $6.36 \mathrm{eV}$, the peak $\mathbf{d}$ is large. From the decomposition analysis shown in Fig. $7, \mu_{z}^{\text {ind }}$ is the main contributor. Since the transition moment is also in the $z$-direction, the peak $\mathbf{d}$ is enhanced as in the case of off-resonance Raman discussed above. Mode $\mathbf{j}$ appears in the spectrum, but it is very weak in the off-resonance condition as discussed above. In the resonance condition, the orbitals involved in the excitation are extended to the $\mathrm{C}-\mathrm{H}$ bonding orbitals, as shown in Fig. 10. The change in the induced dipole moment along this vibration becomes large, which enhances the Raman signal. (a)

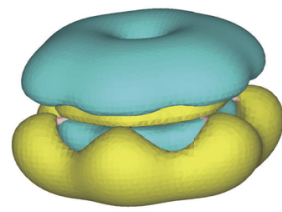

(b)

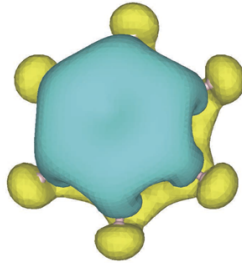

Iso $=1.0 \times 10^{-2}$

(d)

(c)<smiles>CC1C(=O)C(=O)C(=O)C1=O</smiles>

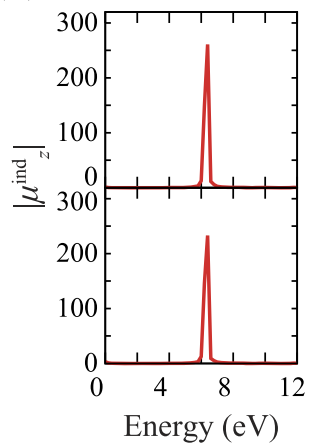

(e)

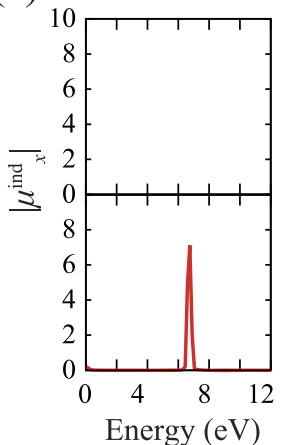

FIG. 10. Fourier-transformed electron density differences for near-field excitations at (a) $6.36 \mathrm{eV}$ and (b) $6.70 \mathrm{eV}$. (c) Benzene at the equilibrium structure and one of its distorted structures. The displaced $\mathrm{C}$ atom is indicated by the red arrow, and its displacement is exaggerated for visibility. The Fourier-transformed induced dipole moments of $\mu_{z}^{\text {ind }}$ and $\mu_{x}^{\text {ind }}$ obtained by near-field excitations at (d) $6.36 \mathrm{eV}$ and (e) $6.70 \mathrm{eV}$, respectively, for the equilibrium (top) and distorted (bottom) structures.

Next, we discuss the near-field Raman at $6.70 \mathrm{eV}$, shown in Figs. 6(g) and 6(h). Comparing the induced electron density difference at the equilibrium geometry [Fig. 10(b)] to that shown in Fig. 4, this excitation is apparently not the $\pi-\pi^{*}$ transition. However, when the molecule has a distorted structure (one of the atomic coordinates is shifted), the $\pi-\pi^{*}$ transition is excited, as shown in Fig. 10(d). Since this could be a vibronic effect, we will call it vibronic resonance Raman for the near field. Let us focus on the peak selectivity, where modes $\mathbf{c}, \mathbf{f}$, and $\mathbf{i}$ are active, although the latter two are very weak. Along the vibrational coordinate in the near field, these three modes can reverse the sign of the dipole moment in the $x y$-plane at the equilibrium geometry $(Q=0)$, while modes $\mathbf{b}$, $\mathbf{d}, \mathbf{h}$, and $\mathbf{j}$ cannot. Therefore, modes $\mathbf{c}, \mathbf{f}$, and $\mathbf{i}$ are active in nearfield Raman, although the dipole moment changes for the two inplane modes ( $\mathbf{f}$ and $\mathbf{i}$ ) are very small compared to the out-of-plane mode c.

These results show that Raman spectra depend on the resonance condition, particularly the nature of the electronic transitions involved in resonance Raman. The present study considered the benzene molecule with high symmetry, but these discussions are also applicable to other molecules. The results here demonstrate that the present approach, which explicitly uses the full spatial structure of the electric field, facilitates the understanding of the selection rule for near-field Raman. 
Let us discuss our method comparing with Barone's formulation for resonance Raman with uniform light ${ }^{56}$ and Luo's generalization for localized light. ${ }^{57}$ Both of these formulations share the same discussion for vibrational states, while electronic transitions are different.

Our protocol is rooted in Jensen's polarizability method. ${ }^{34}$ Mattiat and Luber demonstrated that this method can efficiently provide resonance Raman spectra for a uniform electric field excitation. ${ }^{33}$ The initial state is the ground states for both the electronic and vibrational states, and the final state is the first vibrational excited state in the electronic ground state. Only the fundamental peaks are considered. We do not explicitly consider the vibrational wavefunctions. Our model corresponds to the situation where the electronic ground and excited states share the same equilibrium geometry and the harmonic potential. This is not a bad assumption because the optimized structure for the chosen excited states is almost the same as that of the ground state, where the FranckCondon factor for the vibrational ground states (0-0 transition; $\left.\left\langle v_{0}^{\prime} \mid v_{0}\right\rangle\right)$ is larger than 0.99 and 0.94 for excitation of $\mathrm{z}$ and $\mathrm{x}$ directions, as shown in Fig. 4, respectively. The calculation of the FC factor is done with ezSpectrum 3.0.

Under these conditions, the intermediate states should be vibrational ground or first excited states, otherwise the integral is zero. The FC term is zero since if the intermediate state is $\left|v_{0}^{\prime}\right\rangle$, then the integral with the final state $\left|v_{1}\right\rangle$ vanishes. ${ }^{59}$ In turn, if the intermediate state is $\left|v_{1}^{\prime}\right\rangle$, then the integral with the initial state $\left|v_{0}\right\rangle$ vanishes. In a similar manner, the fourth term of Barone's equation $(10)^{56}$ vanishes. The second and third terms can be survived with $\left\langle v_{1}|Q| v_{0}^{\prime}\right\rangle\left\langle v_{0}^{\prime} \mid v_{0}\right\rangle$ and $\left\langle v_{1} \mid v_{1}^{\prime}\right\rangle\left\langle v_{1}^{\prime}|Q| v_{0}\right\rangle$, respectively. Thus, these two terms can be present in our calculations.

The present Rydberg state accidentally has the similar equilibrium geometry to the ground state, allowing us to neglect the FC term. If this is not the case, one should explicitly consider the vibrational wavefunctions.

It should be worth mentioning that in our calculation, the resonance electronic state is excited for distorted geometries, and that excitation is transition-forbidden for the pristine $D_{6 h}$ structure. This should be related to the vibronic coupling, probably to the Jahn-Teller effect, and we are currently cannot state that this is related to the HT effect because the HT effect refers to the coupling between excited states not between a virtual state and an excited state. Therefore, we did not try to use the term "HT" but just "vibronic." This should be studied in detail in the future.

As for the Cartesian displacements of $0.04 \AA$, this value is chosen for the numerical differentiation, but this displacement surely triggers the excitation to the excited state that is forbidden for the pristine $D_{6 h}$ geometry and can have a substantial effect on the resonance Raman spectrum.

\section{Dependence on tip position}

Near-field Raman spectroscopy, in particular STM-TERS, has been used to map molecular vibrations and studied theoretically. ${ }^{1,19,26,28}$ We also reported resonance STM-TERS imaging experiments, but the molecule was decoupled from the metal surface and the STM tip. This is unlike the previous studies in which the molecule is directly placed on a metal surface, and the distance between the molecule and STM tip is very close, as discussed in the Introduction. Now, we calculate the tip position dependence of the Raman peaks for the off-resonance $(3.0 \mathrm{eV})$ and two on-resonance $(6.36 \mathrm{eV}$ and $6.70 \mathrm{eV})$ conditions discussed above. Figure 11 shows the position of the tip (i.e., the point dipole). Nine points were chosen for the Raman spectra calculations. The distance between adjacent points is $1 \AA$. The angle between the lines connecting A-E and $\mathrm{A}-\mathrm{I}$ is $30^{\circ}$. We chose the same vibrational modes previously reported by Jensen et al., especially for discussing the field gradient effects. ${ }^{20,21}$

\section{Off-resonance}

Figure 12 shows the peak intensities and their decomposition for vibrational modes $\mathbf{b}, \mathbf{c}_{1}, \mathbf{c}_{2}$, and $\mathbf{j}$ as a function of the tip position under the off-resonance condition $(3.0 \mathrm{eV})$. A dependence on the vibrational mode can be observed. The peak intensity for mode $\mathbf{c}_{1}$ decreases as the tip moves away from the molecular center, while mode $\mathbf{j}$ shows the opposite trend. The trend for modes $\mathbf{b}$ and $\mathbf{c}_{2}$ is intermediate between those of modes $\mathbf{c}_{1}$ and $\mathbf{j}$.

The peak intensity of mode $\mathbf{b}$ is maximum at points $\mathrm{B}$ and $\mathrm{F}$ on each line. The main contributors are $\mu_{z}^{\text {ind }}$ and $\mu_{y}^{\text {ind }}$. The $z$ component of the peak monotonically decreases, while the $y$ component has a maximum at point $\mathrm{C}$. When the tip is located at C, $E_{x}^{\mathrm{nf}}$ is symmetric, and thus, $\mu_{x}^{\text {ind }}=0$, whereas $E_{y}^{\text {nf }}$ is asymmetric, and thus, $\mu_{y}^{\text {ind }} \neq 0$. As the tip moves further to point $\mathrm{E}$, the near-field itself becomes weak, and therefore, the peak intensity decreases. This is why $\partial \mu_{y}^{\text {ind }} / \partial Q$ has a maximum at point C. Along line A-I, $E_{x}^{\mathrm{nf}}$ is also asymmetric, and, thus $\mu_{x}^{\text {ind }}$ contributes to the peak intensity. The monotonic decrease in the contribution from $\mu_{z}^{\text {ind }}$ can be explained as follows. When the tip is at the molecular center, all the atoms can contribute to the induced dipole moment. On the other hand, when the tip is away from the center, atoms

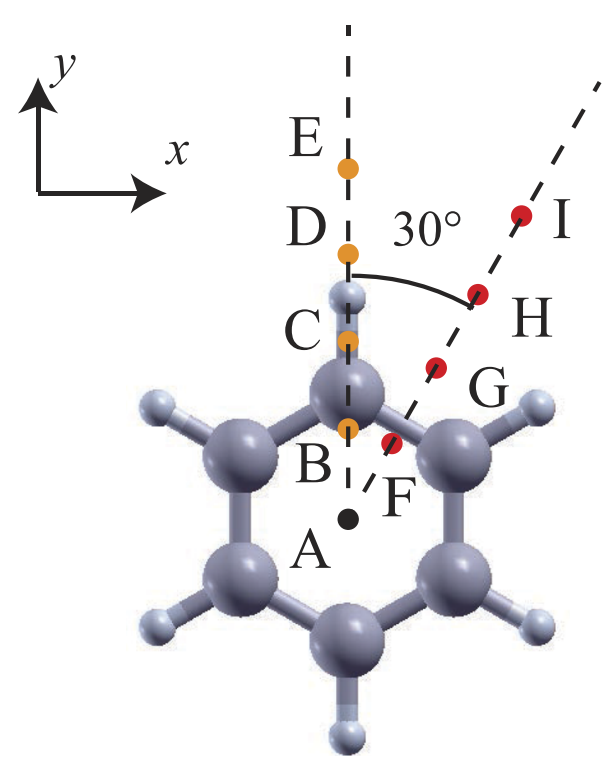

FIG. 11. Positions of the dipole moment for calculating tip position dependence. 
(a) mode $\mathbf{b}$

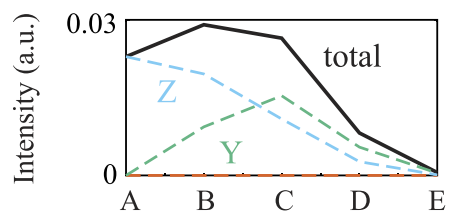

(b) mode $\mathbf{c}_{1}$

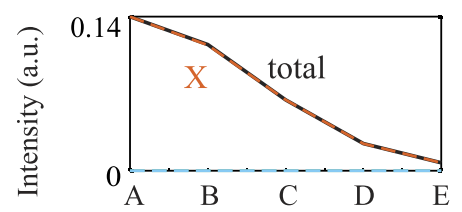

(c) mode $\mathbf{c}_{2}$

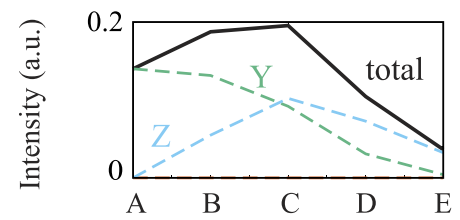

(d) mode $\mathbf{j}$
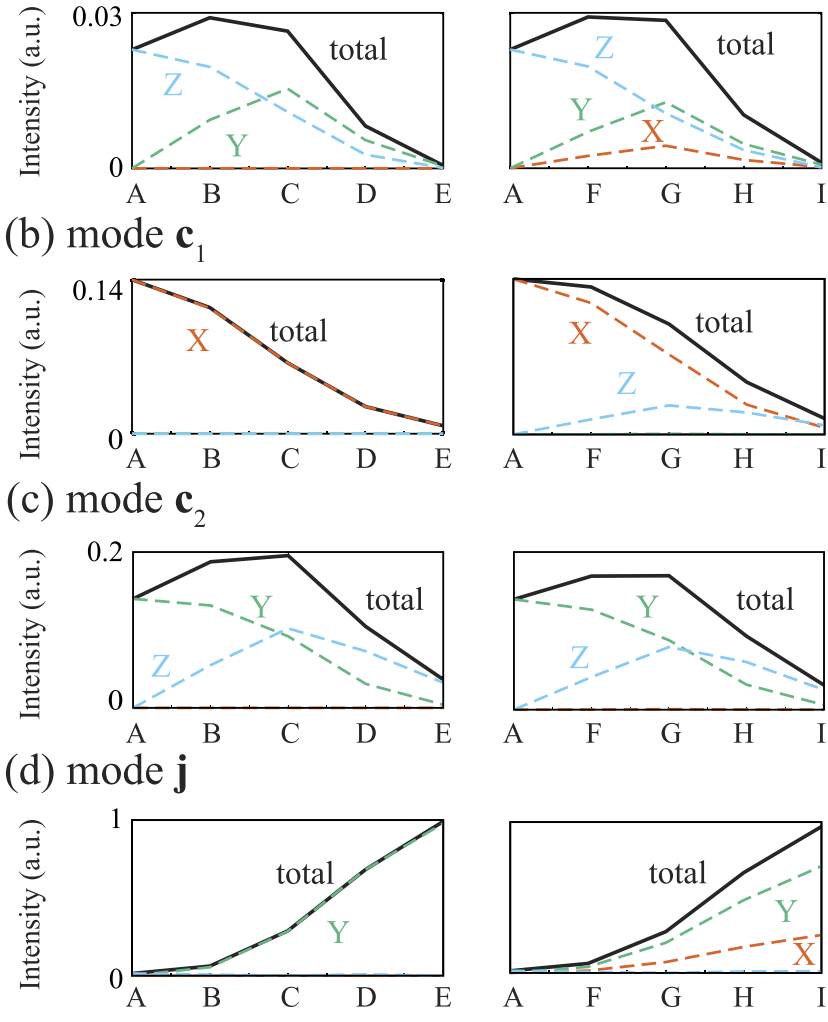

FIG. 12. Raman intensity at each tip position, and the component of $\partial \boldsymbol{\mu} / \partial \boldsymbol{Q}$ under non-resonance condition $(3.00 \mathrm{eV})$. The letters $X, Y$, and $Z$ in the figure represent $\mu_{x}^{\text {ind }}, \mu_{y}^{\text {ind }}$, and $\mu_{z}^{\text {ind }}$, respectively.

far from the tip contribute less, and therefore, the peak intensity decreases.

The peak of mode $\mathbf{c}_{1}$ in Fig. 12(b) is exclusively composed of $\mu_{x}^{\text {ind }}$ on the line A-E. $E_{x}^{\mathrm{nf}}$ is symmetric along this line, and the contribution from $\mu_{x}^{\text {ind }}$ is shown in Fig. 8(b). When the tip is away from the molecular center, the electric field becomes weaker and the peak decreases monotonically. The $z$ component also appears on line A-I. In mode $\mathbf{c}_{1}$ (Fig. 6), most of the motion occurs in four hydrogen atoms. Because the tip position of $G$ is closest to these hydrogen atoms, there is a large induced dipole moment in the $z$ direction, which makes the largest contribution to peak intensity.

The peak of mode $\mathbf{c}_{2}$ is composed of $\mu_{y}^{\text {ind }}$ and $\mu_{z}^{\text {ind }}$. The contribution from $\mu_{y}^{\text {ind }}$ monotonically decreases for the same reason as the contribution from $\mu_{z}^{\text {ind }}$ of mode $\mathbf{b}$. The $\mathrm{z}$ component is maximum at points $\mathrm{C}$ and $\mathrm{G}$ on each line because most of the motion occurs in four atoms on the $y$-axis in mode $\mathbf{c}_{2}$.

Mode $\mathbf{j}$ is the in-plane $\mathrm{C}-\mathrm{H}$ stretching. When the tip is above the center of the molecule, this mode is not Raman active, while it is active in far-field Raman spectroscopy. As the tip moves away from the center of the molecule, the electric field approaches the far field, and thus, the peak intensity monotonically increases.

\section{On-resonance}

Figure 13 shows the Raman spectra at $6.36 \mathrm{eV}$. For mode b, the same discussion given above for off-resonance Raman holds. However, because the excitation is in the $z$ direction, the largest contribution comes from $\mu_{z}^{\text {ind }}$ and very little comes from the $x$ and $y$ components. Mode $\mathbf{c}_{1}$ consists mostly of $\mu_{x}^{\text {ind }}$ as in the case of off-resonance Raman, but in the resonance condition, the maxima are found at points B and F. This is likely because the amount of the induced dipole moment is larger when the tip approaches above the ring edge, where the electron density is larger than that inside the ring. In our method, the amount of excited state induced depends on the field intensity and its spatial variation. For mode $c_{2}$, the $\mathrm{z}$ component monotonically increases on line A-E. The excited state is Rydberg $\pi-\sigma^{*}$, and thus, the contribution from $\mu_{z}^{\text {ind }}$ may become large on the outside of the benzene ring. Mode $\mathbf{j}$ mostly consists of $\mu_{z}^{\text {ind }}$ at $\mathrm{A}$, as discussed in Sec. V B. The maxima are found at $\mathrm{B}$ and $\mathrm{F}$ for the same reason as for mode $\mathbf{c}_{1}$.

\section{(a) mode $\mathbf{b}$}
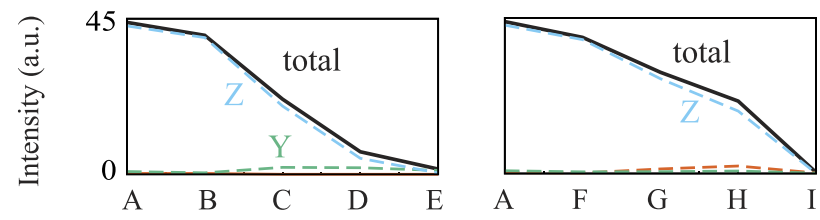

(b) mode $\mathbf{c}_{1}$
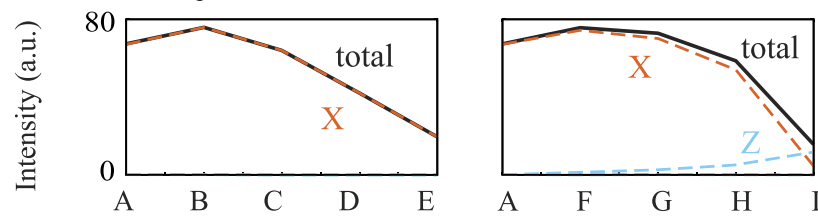

(c) mode $\mathbf{c}_{2}$
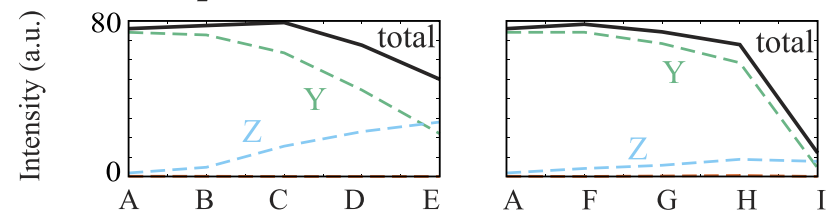

(d) mode $\mathbf{j}$
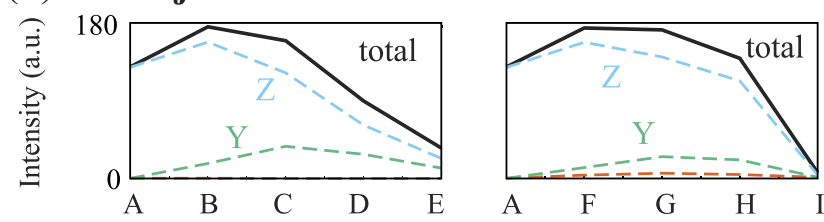

FIG. 13. Raman intensity at each tip position and the component of $\partial \mu / \partial Q$ under resonance condition $(6.36 \mathrm{eV})$. 
Figure 14 shows the Raman spectra at $6.70 \mathrm{eV}$. The behavior is mostly the same as the off-resonance case shown in Fig. 12. The main difference is that the excited state is $\pi-\pi^{*}$ (within the $x y$ plane), and thus, the $x$ and $y$ components are dominant instead of the $z$ component. In mode $\mathbf{c}_{2}$, the contribution from $\mu_{z}^{\text {ind }}$ is as large as $\mu_{y}^{\text {ind }}$. Mode $\mathbf{c}_{2}$ belongs to $\mathrm{E}_{\mathrm{g}}$ symmetry. In far-field spectra, the peak comes from the $y z$ component of the polarizability derivative tensor. Therefore, as the tip moves away from the center of the molecule, $E_{y}^{\mathrm{nf}}$ approaches the far field, and the contribution of $\mu_{z}^{\text {ind }}$ increases. In mode $\mathbf{j}$, the intensity decreases when the tip is far away from the molecule. It can be understood that the amount of excited states becomes small when the tip is distant from the molecule. The decay would be faster for resonance Raman than for off-resonance Raman.

\section{Mapping}

Figure 15 shows the map for these spectra. We have prepared the $2 \mathrm{D}$ images for the modes discussed in tip-position-dependencies. The images show vibrational mode dependencies as obtained via the linear response theory methods. ${ }^{20,60}$ The simulated off-resonance Raman images show vibrational mode dependence as theoretically predicted $^{20,57}$ and later experimentally confirmed. ${ }^{19,61}$ As modes b and $\mathbf{j}$ are $\mathrm{C}_{6}$-symmetric, the map also has the same symmetry, but the bright points are different. Modes $\mathbf{c}_{1}$ and $\mathbf{c}_{2}$ are lower symmetry

\section{(a) mode $\mathbf{b}$}
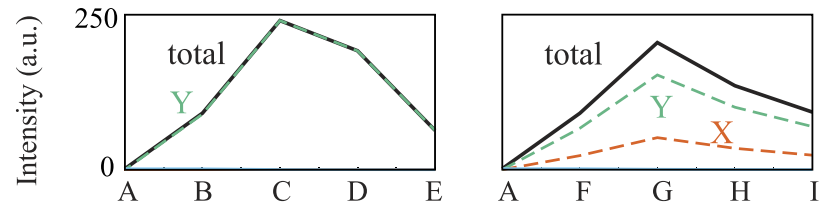

(b) mode $\mathbf{c}_{1}$
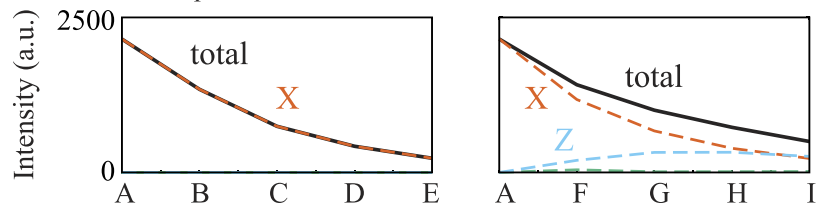

(c) mode $\mathbf{c}_{2}$

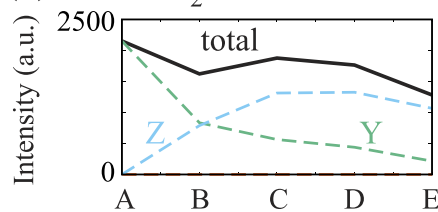

(d) mode j
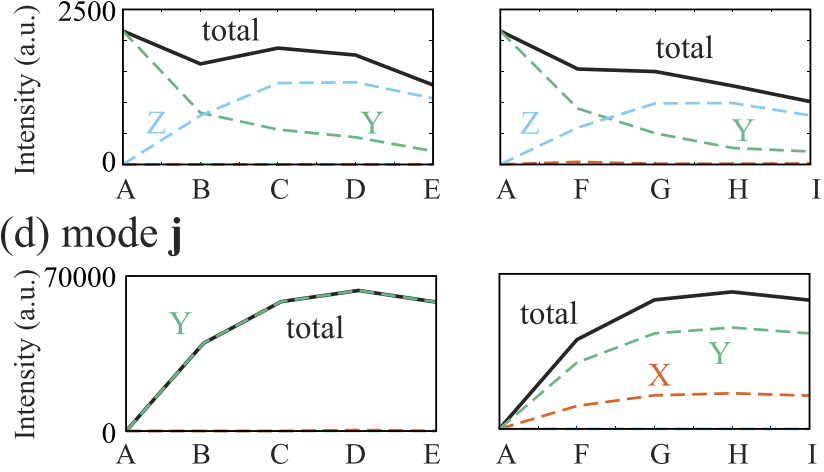

resonance condition $(6.70 \mathrm{eV})$

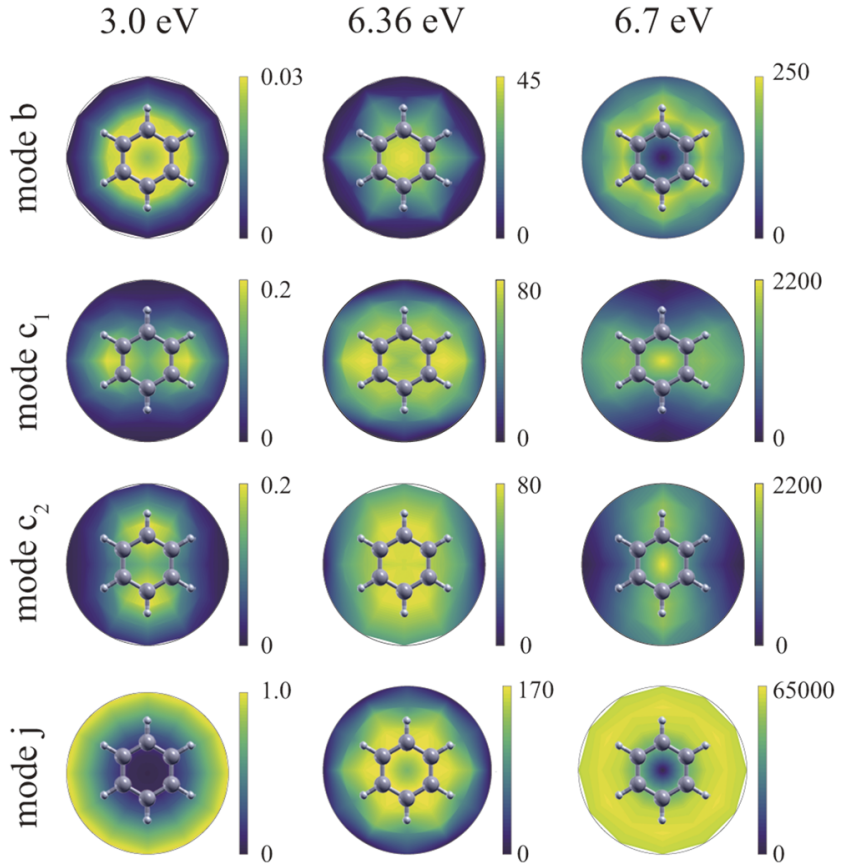

FIG. 15. Off-resonance and on-resonance near-field Raman image mappings of modes $\mathbf{b}, \mathbf{c}_{1}, \mathbf{c}_{2}$, and $\mathbf{j}$.

than $\mathrm{C}_{6}$, and thus, the image also shows bright points different from modes $\mathbf{b}$ and $\mathbf{j}$.

Our model is based on our previous study ${ }^{28}$ in which $E_{x}$ and $E_{y}$ are also important in addition to $E_{z}$. The STM-TERS images obtained theoretically differ from those of previous studies. ${ }^{20,21,54}$ It is worth mentioning that Jensen et al. also indicated that the distance between the molecule and tip can significantly affect the TERS image, i.e., the relative peak intensities. ${ }^{21}$

\section{CONCLUSIONS}

Here, we propose a theoretical method for modeling near-field Raman in the regime beyond the dipole approximation. This method is based on the multipolar Hamiltonian, which can account for the full spatial distribution of the electric field. The method is well suited to our recent STM-TERS experiment that studied the intrinsic molecular properties. The electronic structure calculations are performed under the framework of real-time time-dependent density functional theory, which allows us to treat on- and off-resonance Raman on the same footing. The method was applied to the on- and off-resonance TERS of benzene. The selection rule for off-resonance near-field Raman is well described in terms of the spatial structure of the near field and the molecular vibrations. The on-resonance Raman spectra are well analyzed by considering the transition dipole moments. For resonance near-field Raman, the $\pi-\pi^{*}$ transition of benzene at the equilibrium geometry is forbidden, but it is allowed at distorted structures due to vibronic couplings. Moving the tip 
position can lower the symmetry of the system, which changes the peak intensities as well as the contributing components. Our method can treat the spatial structure of the near field explicitly and provide an intuitive picture of the selection rule for near-field Raman spectroscopy.

\section{ACKNOWLEDGMENTS}

T.I. thanks the financial support provided by the Japan Society for the Promotion of Science (JSPS) KAKENHI (Grant Nos. 20K05412, 20H04652, and 20K05592) and by JST PRESTO (Grant No. JPMJPR20T1). The authors are grateful to the Institute for Quantum Chemical Exploration for the IQCE Fellowships for Young Scientists (M.T.) and for financial support (T.I. and T.T.). This work was also partly supported by the Elements Strategy Initiative of MEXT (Grant No. JPMXP0112101003), the Photo-excitonix Project at Hokkaido University, and JST CREST, Japan (Grant No. JPMJCR1902). The authors thank the Supercomputer Center, Institute for Solid State Physics, University of Tokyo for the use of the facilities and the Research Center for Computational Science, Okazaki, Japan.

\section{DATA AVAILABILITY}

The data that support the findings of this study are available from the corresponding author upon reasonable request.

\section{REFERENCES}

${ }^{1}$ K. Li, N. J. Hogan, M. J. Kale, N. J. Halas, P. Nordlander, and P. Christopher, Nano Lett. 17, 3710 (2017).

${ }^{2}$ E. Kazuma, J. Jung, H. Ueba, M. Trenary, and Y. Kim, Science 360, 521 (2018).

${ }^{3}$ B. Seemala, A. J. Therrien, M. Lou, K. Li, J. P. Finzel, J. Qi, P. Nordlander, and P. Christopher, ACS Energy Lett. 4, 1803 (2019).

${ }^{4} \mathrm{~N}$. Tate and T. Yatsui, Sci. Rep. 9, 18383 (2019).

${ }^{5}$ M. Osawa, K.-I. Ataka, K. Yoshii, and Y. Nishikawa, Appl. Spectrosc. 47, 1497 (1993).

${ }^{6}$ P. L. Stiles, J. A. Dieringer, N. C. Shah, and R. P. Van Duyne, Annu. Rev. Anal. Chem. 1, 601 (2008).

${ }^{7}$ T. Neuman, R. Esteban, D. Casanova, F. J. García-Vidal, and J. Aizpurua, Nano Lett. 18, 2358 (2018).

${ }^{8}$ P. Verma, Chem. Rev. 117, 6447 (2017).

${ }^{9}$ B. Pettinger, P. Schambach, C. J. Villagómez, and N. Scott, Annu. Rev. Phys. Chem. 63, 379 (2012).

${ }^{10}$ F. Shao and R. Zenobi, Anal. Bioanal. Chem. 411, 37 (2019).

${ }^{11}$ R. M. Stöckle, Y. D. Suh, V. Deckert, and R. Zenobi, Chem. Phys. Lett. 318, 131 (2000).

${ }^{12}$ M. S. Anderson, Appl. Phys. Lett. 76, 3130 (2000).

${ }^{13}$ B. Pettinger, G. Picardi, R. Schuster, and G. Ertl, Electrochemistry 68, 942 (2000).

${ }^{14}$ Z. Zhang, M. Sun, P. Ruan, H. Zheng, and H. Xu, Nanoscale 5, 4151 (2013).

${ }^{15}$ E. J. Ayars, H. D. Hallen, and C. L. Jahncke, Phys. Rev. Lett. 85, 4180 (2000).

${ }^{16}$ J. Steidtner and B. Pettinger, Phys. Rev. Lett. 100, 236101 (2008).

${ }^{17}$ C. Chen, N. Hayazawa, and S. Kawata, Nat. Commun. 5, 3312 (2014).

${ }^{18}$ R. Zhang, Y. Zhang, Z. C. Dong, S. Jiang, C. Zhang, L. G. Chen, L. Zhang, Y. Liao, J. Aizpurua, Y. Luo, J. L. Yang, and J. G. Hou, Nature 498, 82 (2013).

${ }^{19}$ J. Lee, K. T. Crampton, N. Tallarida, and V. A. Apkarian, Nature 568, 78 (2019).
${ }^{20}$ P. Liu, D. V. Chulhai, and L. Jensen, ACS Nano 11, 5094 (2017).

${ }^{21}$ X. Chen, P. Liu, Z. Hu, and L. Jensen, Nat. Commun. 10, 2567 (2019).

${ }^{22}$ S. Duan, G. Tian, Y. Ji, J. Shao, Z. Dong, and Y. Luo, J. Am. Chem. Soc. 137, 9515 (2015).

${ }^{23}$ S. Duan, G. Tian, Z. Xie, and Y. Luo, J. Chem. Phys. 146, 194106 (2017).

${ }^{24}$ Z. Xie, S. Duan, G. Tian, C.-K. Wang, and Y. Luo, Nanoscale 10, 11850 (2018).

${ }^{25}$ S. Duan, Z. Xie, G. Tian, and Y. Luo, J. Phys. Chem. Lett. 11, 407 (2020).

${ }^{26}$ K. Fiederling, M. Abasifard, M. Richter, V. Deckert, S. Gräfe, and S. Kupfer, Nanoscale 12, 6346 (2020).

${ }^{27}$ J. Repp, G. Meyer, S. M. Stojković, A. Gourdon, and C. Joachim, Phys. Rev. Lett. 94, 026803 (2005).

${ }^{28}$ R. B. Jaculbia, H. Imada, K. Miwa, T. Iwasa, M. Takenaka, B. Yang, E. Kazuma, N. Hayazawa, T. Taketsugu, and Y. Kim, Nat. Nanotechnol. 15, 105 (2020).

${ }^{29}$ T. Iwasa, M. Takenaka, and T. Taketsugu, J. Chem. Phys. 144, 124116 (2016).

${ }^{30}$ M. Takenaka, T. Taketsugu, and T. Iwasa, J. Chem. Phys. 152, 164103 (2020).

${ }^{31}$ T. Iwasa and K. Nobusada, Phys. Rev. A 80, 043409 (2009).

${ }^{32}$ M. Thomas, F. Latorre, and P. Marquetand, J. Chem. Phys. 138, 044101 (2013).

${ }^{33}$ J. Mattiat and S. Luber, J. Chem. Phys. 149, 174108 (2018).

${ }^{34}$ L. Jensen, L. L. Zhao, J. Autschbach, and G. C. Schatz, J. Chem. Phys. 123, 174110 (2005).

${ }^{35}$ X. Andrade, J. Alberdi-Rodriguez, D. A. Strubbe, M. J. T. Oliveira, F. Nogueira, A. Castro, J. Muguerza, A. Arruabarrena, S. G. Louie, A. Aspuru-Guzik, A. Rubio, and M. A. L. Marques, J. Phys.: Condens. Matter 24, 233202 (2012).

${ }^{36}$ K. Iida, M. Noda, K. Ishimura, and K. Nobusada, J. Phys. Chem. A 118, 11317 (2014).

${ }^{37}$ E. B. Wilson, P. C. Cross, and J. C. Decius, Molecular Vibrations: The Theory of Infrared and Raman Vibrational Spectra (Dover Publications, Inc., NY, 1980).

${ }^{38}$ D. P. Craig and T. Thirunamachandran, Molecular Quantum Electrodynamics: An Introduction to Radiation-Molecule Interactions (Dover, Mineola, NY, 1998).

${ }^{39}$ A. D. Buckingham, in Advances in Chemical Physics, edited by J. O. Hirschfelder (Wiley Online Library, 2007), Vol. 12, pp. 107-142.

${ }^{40} \mathrm{X}$. Andrade, D. Strubbe, U. De Giovannini, A. H. Larsen, M. J. T. Oliveira, J. Alberdi-Rodriguez, A. Varas, I. Theophilou, N. Helbig, M. J. Verstraete, L. Stella,

F. Nogueira, A. Aspuru-Guzik, A. Castro, M. A. L. Marques, and A. Rubio, Phys. Chem. Chem. Phys. 17, 31371 (2015).

${ }^{41}$ J. P. Perdew, K. Burke, and M. Ernzerhof, Phys. Rev. Lett. 77, 3865 (1996).

${ }^{42}$ J. P. Perdew, K. Burke, and M. Ernzerhof, Phys. Rev. Lett. 78, 1396 (1997).

${ }^{43}$ D. R. Hamann, Phys. Rev. B 88, 085117 (2013).

${ }^{44}$ K. Yabana and G. F. Bertsch, Phys. Rev. B 54, 4484 (1996).

${ }^{45}$ R. Sinha-Roy, P. García-González, X. López Lozano, R. L. Whetten, and H.-C. Weissker, J. Chem. Theory Comput. 14, 6417 (2018),

${ }^{46}$ J. Jornet-Somoza and I. Lebedeva, J. Chem. Theory Comput. 15, 3743 (2019).

${ }^{47}$ L. Meng, Z. Yang, J. Chen, and M. Sun, Sci. Rep. 5, 9240 (2015).

${ }^{48}$ R. Ahlrichs, M. Bär, M. Häser, H. Horn, and C. Kölmel, Chem. Phys. Lett. 162, 165 (1989).

${ }^{49}$ R. A. Kendall, T. H. Dunning, and R. J. Harrison, J. Chem. Phys. 96, 6796 (1992).

${ }^{50}$ Y. Li, J. Wan, and X. Xu, J. Comput. Chem. 28, 1658 (2007).

${ }^{51}$ J. Lorentzon, P. Å. Malmqvist, M. Fülscher, and B. O. Roos, Theor. Chim. Acta 91, 91 (1995).

${ }^{52}$ J. Neugebauer, M. Reiher, C. Kind, and B. A. Hess, J. Comput. Chem. 23, 895 (2002).

${ }^{53}$ D. Rappoport and F. Furche, J. Chem. Phys. 126, 201104 (2007).

${ }^{54} \mathrm{D}$. V. Chulhai and L. Jensen, J. Phys. Chem. C 117, 19622 (2013).

${ }^{55}$ M. Takase, H. Ajiki, Y. Mizumoto, K. Komeda, M. Nara, H. Nabika, S. Yasuda, H. Ishihara, and K. Murakoshi, Nat. Photonics 7, 550 (2013). 
${ }^{56}$ F. Santoro, C. Cappelli, and V. Barone, J. Chem. Theory Comput. 7, 1824 (2011).

${ }^{57}$ S. Duan, G. Tian, and Y. Luo, J. Chem. Theory Comput. 12, 4986 (2016).

${ }^{58}$ V. Mozhayskiy and A. Krylov, ezSpectrum, http://iopenshell.usc.edu/ downloads.
${ }^{59}$ D. A. Long, The Raman Effect: A Unified Treatment of the Theory of Raman Scattering by Molecules (John Wiley \& Sons, Ltd., Chichester, 2002).

${ }^{60}$ Z. Xie, S. Duan, C.-K. Wang, and Y. Luo, J. Phys. Chem. C 123, 11081 (2019).

${ }^{61}$ Y. Zhang, B. Yang, A. Ghafoor, Y. Zhang, Y.-F. Zhang, R.-P. Wang, J.-L. Yang, Y. Luo, Z.-C. Dong, and J. G. Hou, Natl. Sci. Rev. 6, 1169 (2019). 